\title{
A Systemically Administered Neurotensin Agonist Blocks Disruption of Prepulse Inhibition Produced by a Serotonin-2A Agonist
}

\author{
David Feifel*,', Gilia Melendez' and Paul D Shilling' \\ 'Department of Psychiatry, University of California, San Diego, CA, USA
}

Prepulse inhibition (PPI) of the startle reflex can be disrupted by drugs that act as agonists at the serotonin (5-HT) 2A receptor, such as $\mathrm{DOI}$, and this effect is blocked by drugs that inhibit 5-HT2A transmission. We tested the effects of systemic administration of PDI49/63, a neurotensin agonist, on DOI-induced disruption of PPI in Sprague-Dawley rats. PDI49 63 completely and dose dependently blocked the PPI deficits produced by DOI. These findings suggest that, in addition to their established ability to inhibit dopamine transmission, neurotensin agonists may also inhibit 5-HT2A transmission, a pharmacological feature associated with atypical antipsychotic drugs. Neuropsychopharmacology (2003) 28, 65I-653. doi:I 0.1038/sj.npp. 1300083

\section{INTRODUCTION}

Prepulse inhibition (PPI) of the acoustic startle reflex is the reduction in the startle response when the startle-eliciting stimulus is immediately preceded by a weak stimulus. PPI, an operational measure of sensorimotor gating, is deficient in schizophrenia patients (Geyer et al, 2001).

Deficits in PPI can be produced in rats by a number of pharmacologically distinct 'psychotomimetic' compounds (see Geyer et al, 2001 for a review). Typical and atypical antipsychotics reverse PPI deficits produced by dopamine agonists such as amphetamine and apomorphine. The mechanism implicated has been blockade of dopamine-2 (D2) receptors. In contrast, PPI disruption produced by noncompetitive NMDA antagonists such as phencyclidine (PCP) or dizocilpine is antagonized by atypical but not typical antipsychotics and blockade of serotonin (5-HT) 2A and/or $\alpha-1$ noradrenergic, but not D2 receptors, has been implicated in this effect. Disruption by the selective 5-HT2A agonist and hallucinogen, 1-(2,5-dimethoxy-4-iodophenyl)2 -aminopropane hydrochloride (DOI) is also preferentially blocked by atypical antipsychotics and the mechanism implicated is blockade of 5-HT2A receptors since a 5-HT2A antagonist but neither a 5-HT2C antagonist nor haloperidol effectively block DOI's effect on PPI (Geyer et al, 2001).

\footnotetext{
*Correspondence: David Feifel, Department of Psychiatry, UCSD Medical Center, 200 West Arbor Drive, San Diego, CA 92103-8218, USA, Tel: + 619543 2485, Fax: + 619543 3738,

E-mail: dfeifel@ucsd.edu

Received 4 June 2002; revised 23 September 2002; accepted 25 September 2002

Online publication: 10 October 2002 at http://www.acnp.org/citations/ Npp I01002404
}

The neuropeptide neurotensin appears to inhibit dopamine function and produce preclinical effects similar to antipsychotics (Kinkead and Nemeroff, 2002) suggesting that neurotensin agonists may have potential as antipsychotic drugs. Supporting this notion, we have reported that PD149163, a modified neurotensin (8-13) analog (Lys(CH2NH)Lys-Pro-Trp-tLe-Leu-OEt) (Wustrow et al, 1995) that crosses the blood brain barrier, antagonizes amphetamine-induced disruption of PPI after systemic administration (Feifel et al, 1999). In addition, we found that PD149163 antagonizes PPI deficits produced by the noncompetitive NMDA antagonist dizocilpine (Feifel et al, 1999), suggesting that this compound modulates 5-HT2A and/or $\alpha-1$ adrenergic transmission (Geyer et al, 2001). There is evidence that neurotensin regulates 5 -HT brain systems (Heaulme et al, 1998), therefore we hypothesized that PD149163 may produce antagonism of dizocilpineinduced disruption of PPI by blockade of 5-HT2A transmission. In order to test this hypothesis we investigated whether PD149163 could antagonize PPI disruption produced by DOI, a selective 5-HT2A agonist.

\section{METHODS}

All experimental procedures were conducted in accordance with the University of California, San Diego guidelines for animal care and experimentation. A total of 34 male Sprague-Dawley rats (250-300 g at testing, Harlan Laboratories, San Diego) were housed under a $12 \mathrm{~h}: 12 \mathrm{~h}$ light: dark schedule. On test days they were administered subcutaneous (s.c.) injections of 0 (saline), $0.01,0.1$, or $1 \mathrm{mg} / \mathrm{kg}$ of PD149163 (Courtesy of NIMW's chemical synthesis and drug supply programme, SRI International, 
Menlo Park, CA). After 30 min they were injected s.c. with either saline or $0.5 \mathrm{mg} / \mathrm{kg}$ DOI (Sigma Chemicals, St Louis, MO). Animals were tested in startle chambers (San Diego Instruments, San Diego, CA) 20 min later. After 1 week, animals were tested a second time during which treatment and testing procedures were the same except that rats that received DOI on the first test day received saline on the second test day and vice versa. All testing occurred during the light phase of the rats' circadian illumination schedule.

Once placed in startle chambers each rat had a 5-min acclimation period. A $65-\mathrm{dB}$ background noise was continuously present throughout the session. The acclimation was followed by a 15-min PPI test session during which rats were presented with $40-\mathrm{ms} 120 \mathrm{~dB}$ startle pulses without a prepulse, or pulses preceded $100-\mathrm{ms}$ by a prepulse of either 4,8 , or $12 \mathrm{~dB}$ above background. These four types of active stimuli were presented in pseudorandom order along with no-sound trials with an average of $15 \mathrm{~s}$ separating them.

A startle response was recorded for all stimuli presentations. PPI for each animal was calculated as a percentage of the pulse-alone startle magnitude using the following formula: (1-(startle magnitude after prepulse-pulse pair/startle magnitude after pulse only))100. PPI data was analyzed using a repeated measures ANOVA with PD149163 dose as a between-subject factor and DOI treatment and prepulse intensity as within-subject factors. Significant effects were followed by post hoc pair-wise comparisons of individual treatment groups using Bonferroni corrected $t$-tests.

\section{RESULTS}

There was no main effect of PD149163 but there was a significant main effect of DOI as it significantly disrupted
PPI $(\mathrm{F}(1,30)=56.1, P<0.001)$. There was a significant main effect of prepulse intensity on percent PPI, reflected in more intense prepulses producing greater PPI $(F(2,60)=80.3$, $P<0.0001)$. However, there was not a significant prepulse $\times$ DOI interaction, or prepulse $\times$ PD149163 interaction or prepulse $\times$ DOI $\times$ PD149163 interaction. Therefore, the PPI data presented in the figure (main graph) is the mean of the PPI values produced by each of the individual prepulse intensities. There was a significant $\mathrm{DOI} \times \mathrm{PD} 149163$ interaction $(\mathrm{F}(3,30)=7.3, P=0.001)$ and the data revealed that the highest dose of PD149163 reversed the DOI-induced disruption of PPI. Compared to rats that did not receive DOI (saline), DOI-treated rats had significantly decreased PPI in the group that did not receive PD149163 (saline) $(P<0.01)$ and in the groups that received $0.01 \mathrm{mg} / \mathrm{kg} \quad(P<0.001)$ and $0.1 \mathrm{mg} / \mathrm{kg}$ $(P<0.05)$, but not $1 \mathrm{mg} / \mathrm{kg}$ PD149163. PPI exhibited by rats receiving DOI and $1 \mathrm{mg} / \mathrm{kg}$ PD149163 was significantly greater than PPI exhibited by rats receiving DOI and saline $(P<0.01)$.

There were no significant main or interaction effects of DOI or PD149163 on startle magnitude (Figure 1).

\section{DISCUSSION}

To date, all drugs that have demonstrated a robust ability to antagonize DOI-induced disruption of PPI have been compounds with potent 5-HT2A antagonism, including the atypical antipsychotic, risperidone, and the selective 5HT2A antagonists, MDL100907 and ketanserin. In contrast, drugs that are not strong 5-HT2A antagonists, including haloperidol, the selective D2 antagonist raclopride, the 5-HT2C antagonist, SDZ SER-082, and the $\beta$-adrenergic

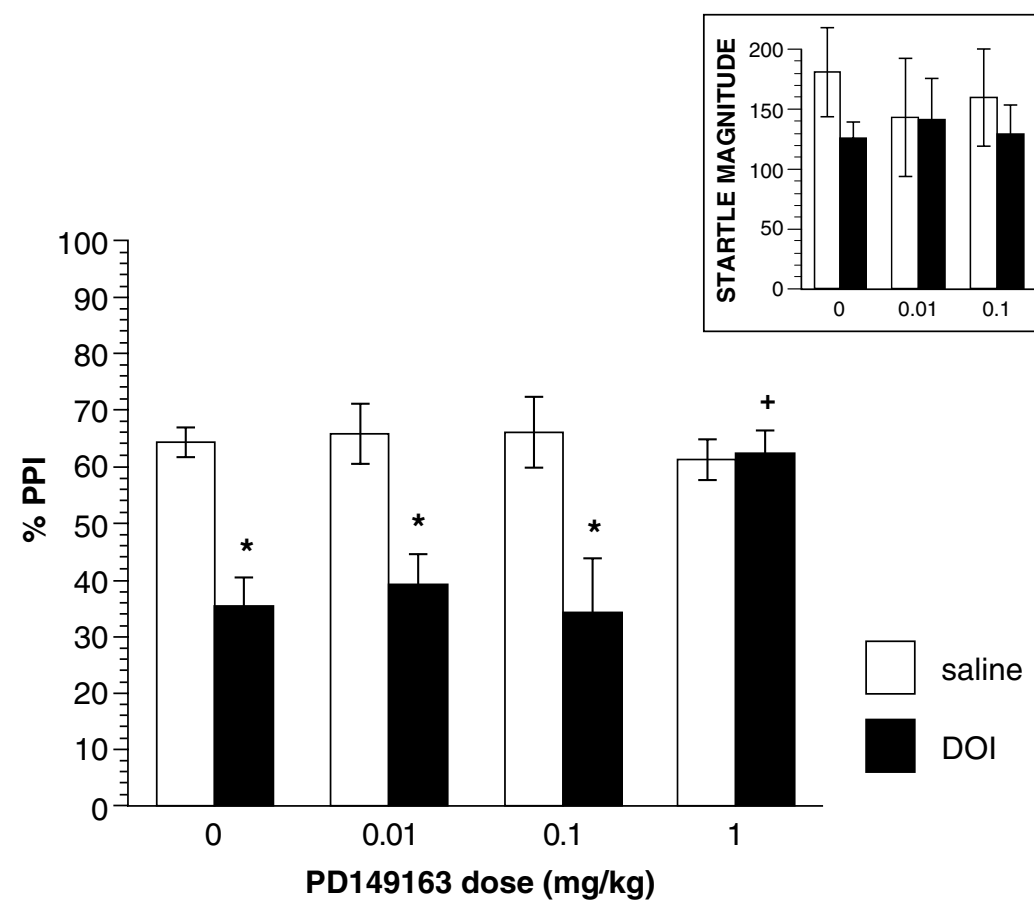

Figure I The effect of PDI49I63 on PPI (main) and startle magnitude (insert) in rats receiving saline or DOI. PPI data represents the average of PPI produced by the three different prepulse intensities. *Represents significantly lower $(P<0.05)$ than corresponding non-DOI treatment. +Represents significantly greater $(P<0.01)$ than rats receiving $0 \mathrm{mg} / \mathrm{kg}$ dose of PDI49I63 and the same DOI treatment. 
15-HT1 receptor antagonist, propanolol, have failed to exhibit robust antagonism of DOI's effects on PPI (Geyer et al, 2001). In this respect, PD149163's ability to block DOI-induced disruption of PPI but not affect baseline PPI or startle magnitude supports our hypothesis that PD149163 may antagonize 5-HT2A transmission.

The modulation of 5-HT systems by neurotensin has not been extensively studied, however neurotensin has been shown to stimulate the release of 5-HT in the brain (Heaulme et al, 1998). Neither neurotensin nor PD149163 is known to have a strong affinity for 5-HT2A receptors. These compounds could alter 5-HT2A transmission via activation of neurotensin receptors that, in turn, modulate 5-HT2A transmission at the receptor level or further downstream. Evidence suggests that DOI-induced disruption of PPI is mediated by 5-HT2A receptors in the ventral pallidum (Sipes and Geyer, 1997), a site where neurotensin receptors have been localized (Alexander and Leeman, 1998). The current evidence that PD149163 modulates 5-HT2A transmission is notable since previous interest in neurotensin agonists as potential antipsychotics was based exclusively on evidence that they inhibited dopamine transmission. Inhibition of both 5-HT2A and D2 transmission is considered the pharmacological profile that distinguishes atypical antipsychotics from the more D2 selective typical antipsychotics (Meltzer, 1999).

\section{ACKNOWLEDGEMENTS}

This work was supported by an NIMH Grant (MH62451) to DF.

\section{REFERENCES}

Alexander MJ, Leeman SE (1998). Widespread expression in adult rat forebrain of mRNA encoding high-affinity neurotensin receptor. J Comp Neurol 402: 475-500.

Feifel D, Reza TL, Wustrow DJ, Davis MD (1999). Novel antipsychotic-like effects on prepulse inhibition of startle produced by a neurotensin agonist. J Pharmacol Exp Ther 288: 710-713.

Geyer MA, Krebs-Thomson K, Braff DL, Swerdeow NR (2001). Pharmacological studies of prepulse inhibition models of sensorimotor gating deficits in schizophrenia: a decade in review. Psychopharmacology (Berl) 156: 117-154.

Heaulme M, Leyris R, Soubrie P, Le Fur G (1998). Stimulation by neurotensin of $(3 \mathrm{H}) 5$-hydroxytryptamine $(5 \mathrm{HT})$ release from rat frontal cortex slices. Neuropeptides 32: 465-471.

Kinkead B, Nemeroff CB (2002). Neurotensin: an endogenous antipsychotic? Curr Opin Pharmacol 2: 99-103.

Meltzer HY (1999). The role of serotonin in antipsychotic drug action. Neuropsychopharmacology 21(Suppl. 2): 106S-115S.

Sipes TE, Geyer MA (1997). DOI disrupts prepulse inhibition of startle in rats via 5 -HT2A receptors in the ventral pallidum. Brain Res 761: 97-104.

Wustrow DJ, Davis MD, Akunne HC, Corbin AE, Wiley JN, Wise LD, Weffner TC (1995). Reduced amide bond neurotensin 8-13 mimetics with potent in vivo activity. Bioorg Med Chem Lett 5: $998-1002$. 